\title{
Research Progress on the Temperature Field of Steel-Concrete Composite Bridge
}

\author{
Cheng Liu ${ }^{1 *}$, Jiansheng Fan $^{2}$, and Lifa Xiong ${ }^{3}$ \\ ${ }^{1}$ Research Institute of Highway (RIOH), Ministry of Transport, Beijing, 100088, China \\ ${ }^{2}$ Department of Civil Engineering, Tsinghua University, Beijing, 100084, China \\ ${ }^{3}$ China-Road Transportation Verification \& Inspection Hi-Tech Co., Ltd., Beijing, 100088, China
}

\begin{abstract}
The research of the temperature effect of steel-concrete composite bridges is of great significance to ensure the safety and durability. The latest research progress in the field of composite bridge temperature field is introduced, including the temperature field test of indoor and outdoor composite bridge, the fine finite element model of bridge temperature field and temperature distribution law of composite bridge. The study proposes two vertical temperature distribution modes of composite bridges under the action of sunlight, which can be used as reference for composite bridge design.
\end{abstract}

\section{Introduction}

Under normal working conditions, the bridge is affected by environmental factors such as solar radiation, seasonal climate change, rain and snow, cold wave, and temperature difference between day and night. The combination of these environmental factors can lead to complex and variable temperature distributions in the bridge structure and hence cause unpredictable temperature deformation and stress.

Studies have shown that [1-5], the temperature field distribution of the composite bridge and the temperature distribution of the concrete or steel beam of a single material are quite different. The thermal conductivity (heat transfer capacity) of steel is much larger than that of concrete, while the specific heat capacity (heat storage capacity) is smaller than that of concrete. Therefore, when the outside temperature changes rapidly, the temperature of the steel beam quickly approaches the ambient temperature, while the temperature of the concrete changes slowly. The unsynchronized temperature changes between the two will cause a significant temperature difference between the steel beam and the concrete bridge deck, causing selfbalancing stress and additional deformation of the structure in the composite beam section. If the additional deformation of the structure is restrained, significant constrained stress will generate in the composite bridge. In some severe cases, the bridge deck may be cracked and the support may be lifted.
At present, China is at the peak of bridge construction. Steel-concrete composite bridges have been widely used for their excellent mechanical properties, construction performance and economics. The research on composite bridges is in full swing. However, domestic steelconcrete research on the temperature field and temperature effect of composite bridges is still lagging behind. The design of steel-concrete composite bridges used by engineers is still based on the theory of temperature field of original concrete bridges. Research on temperature field and temperature effects is very necessary. This article will mainly introduce the latest research work done by the authors' team in recent years on the temperature field of steel-concrete composite bridges, including: (1) temperature field test technology; (2) refined numerical model of composite bridge temperature field; (3) The distribution law of the temperature field of the composite bridge is explored.

\section{Temperature field test technology}

Real bridge testing and model testing are two methods of temperature field testing, but the former is limited be specific bridge engineering examples, whereas the later have greater flexibility in test specimen design, measurement options, and test conditions. This section describes the temperature field test of the composite beam segment model.

\subsection{Test Setup}




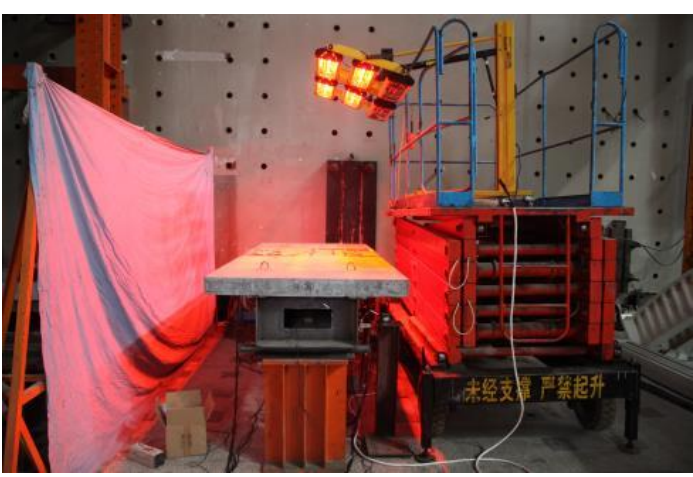

(a) Indoor radiation test

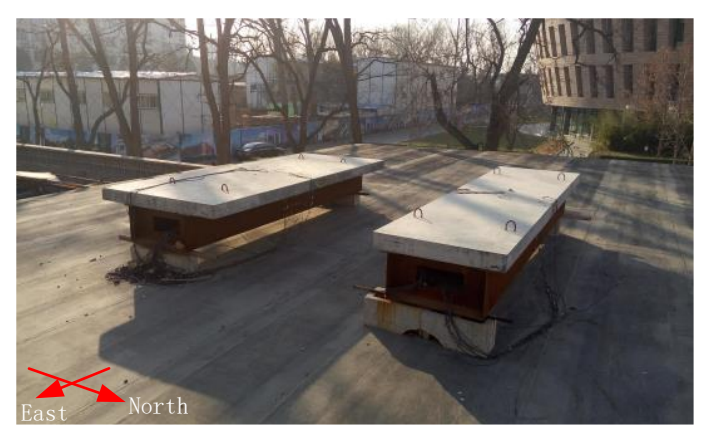

(b) Outdoor test

Figure 1 Illustration of the indoor and outdoor thermal test

A set of two steel-concrete composite beam specimens were designed. The indoor radiation test and the outdoor test were respectively carried out, as shown in Figure 1.

(1) The indoor radiation test is carried out in a test laboratory. The specimens are arranged according to a simple-supported beam, and the distance between the supports is $2800 \mathrm{~mm}$. In the test, a radiation lamp was placed on the side of the specimen at the mid-span position. The radiation lasts for 4 hours and then the lamp was turned off to let the specimen cool for 2 hours. The whole process of temperature rise and fall was regarded as one working condition. Each specimen was loaded in 4 working conditions, in which the radiation angles were set to $80^{\circ}, 60^{\circ}, 30^{\circ}$ and $0^{\circ}$, respectively. The variables measured in the laboratory test included the temperatures, strains and displacements of the specimen.

(2) In the outdoor test, the specimen is placed on the roof of a building to avoid shades from surroundings. The tested beam is placed longitudinally in the east-west direction.

\subsection{Test Result}

The cross-section temperature distribution of the test piece during temperature rise and cooling was extracted, where the uniform temperature part was removed to obtain the vertical temperature differences under different radiation angles as shown in Figure 2. Taking the temperature measurement data of a certain day (September 25th) in the outdoor test as an example, the temperature of each test point of the cross section of the test piece is extracted at the same time, and the timetemperature change of the cross-section temperature distribution is plotted, as shown in Figure 3.

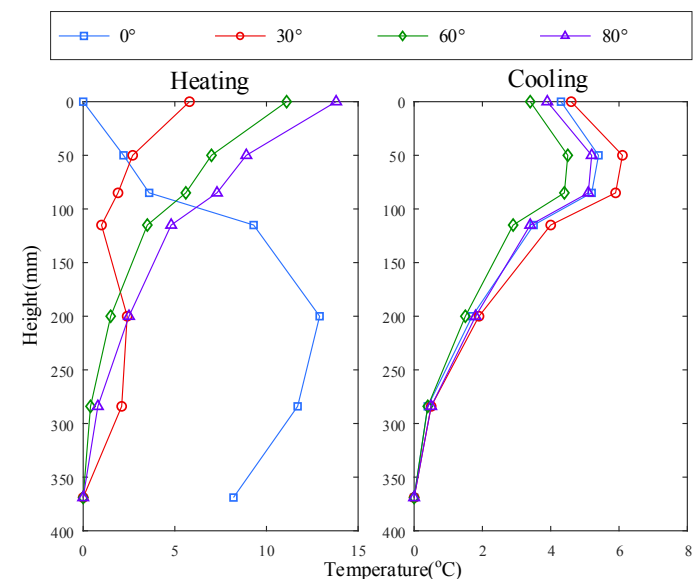

Figure 2 Vertical temperature distribution under different radiation angles (indoor test)

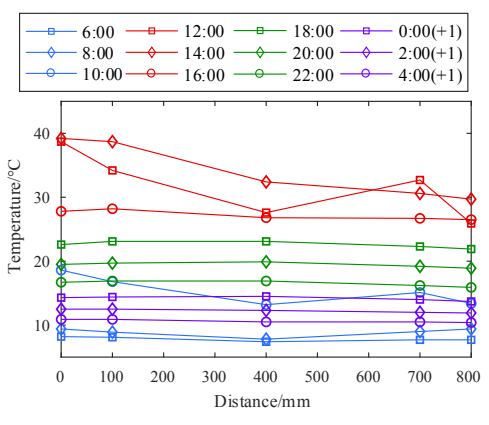

(a) top of the concrete deck

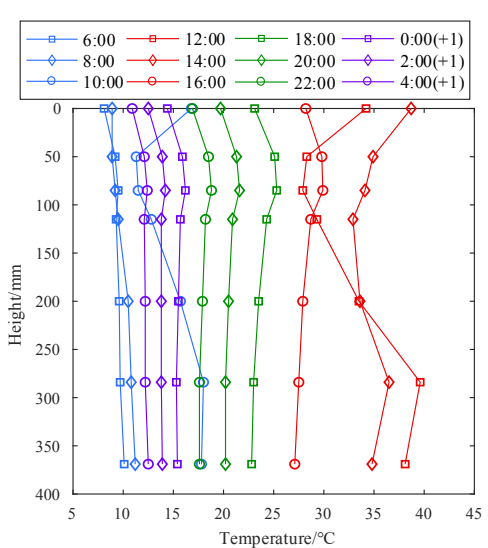

(b) radiation side

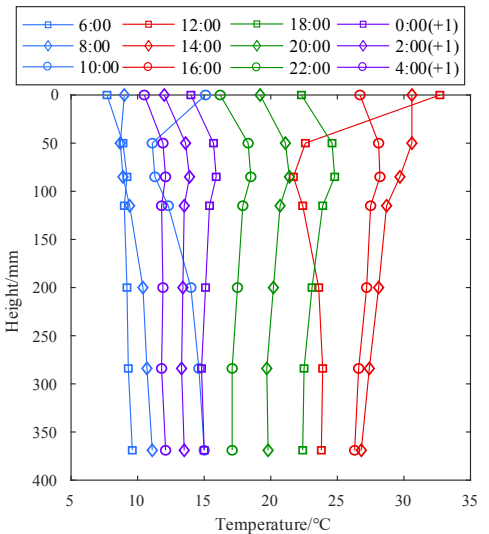

(c) shade side

Figure 3 Temperature distribution of the specimens in the outdoor test 
The temperature distribution results of the composite beam specimens are analysed:

(1) In the outdoor environment, the lateral temperature gradient of the concrete top surface is small, and most of the time can be regarded as uniform distribution.

(2) The vertical temperature distribution of the composite beam basically shows two distribution modes, heating and cooling. When the temperature rises, the temperature shows a nonlinear decrease from top to bottom; when the temperature is lowered, the temperature of the top and bottom surfaces is close to the ambient temperature, where the temperature inside the concrete is the highest.

(3) When the outer flange end of the concrete flange is short, the lower half of the steel beam web is heated by solar radiation, which can produce significant lateral temperature effects.

\section{Refined numerical model}

A bridge is in an atmospheric environment under normal working conditions and is subject to various environmental factors such as solar radiation, seasonal climate change, temperature difference between day and night, rain and snow, and cold wave. Among these environmental factors, solar radiation and seasonal climate change are two key factors affecting the temperature field of the bridge. The former determines the daily temperature cycle of the bridge on a daily basis, while the latter determines the seasonal temperature cycle of the bridge on an annual basis. The prerequisite for accurate simulation of the sunshine temperature field is to accurate model the ambient air temperature and solar radiation.

\subsection{Daily Ambient Temperature}

The accurate description of the weather system is still a unsolved problem. In the temperature field simulation of the composite bridge, the ambient temperature is equivalent to a boundary condition in the numerical model. The model should be as simple as possible, and at the same time reflect the basic lifting law of temperature change. According to the basic law of the change of ambient temperature under ideal sunny day, this paper proposes a daily temperature model to describe the change law of sunny ambient temperature, as shown in Figure 4.

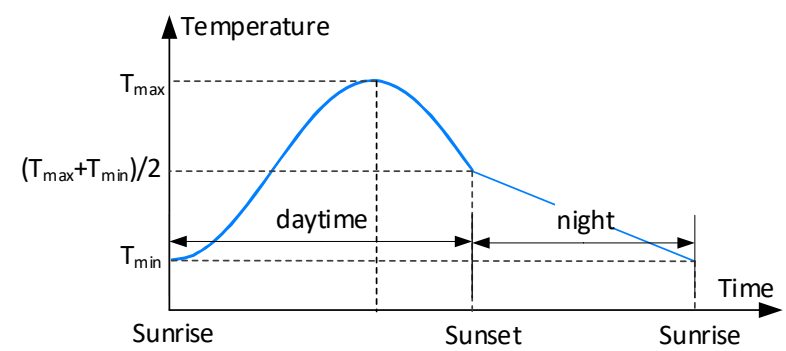

Figure 4 Daily ambient temperature model

\subsection{Solar Radiation}

The solar radiation model is the key to accurately simulating the daily temperature cycle of the bridge. A complete solar radiation calculation model consists of two parts: (1) calculating the relative position of the sun relative to a plane on the ground at any date and time; (2) considering the refraction and scattering of solar radiation by the Earth's atmosphere. The calculation of the former belongs to astronomical geography calculation and the theoretical difference of different solar radiation models mainly lies in the latter.

From the perspective of the simulation of the temperature field of the bridge, it is necessary to select a model that can reflect the solar radiant heat effect and require the input parameters to be as simple as possible. In this paper, the Duffie model [6] was chosen as the basis for the calculation of solar radiation. The model is suitable for the calculation of solar radiation in sunny days, in which the refraction and scattering effects of the atmosphere are quantified by atmospheric transparency. The amount of radiation on any surface is decomposed into three parts: direct radiation, scattered radiation and ground reflected radiation.

\subsection{Numerical Model}

The finite element simulation of transient thermal conduction of bridge temperature field problem is carried out by using the finite element software ABAQUS. The thermal boundary conditions in the finite element model of the temperature field include solar radiation boundary, convective heat transfer and external radiation heat dissipation. The convective heat transfer and the desired radiative heat dissipation boundary are determined by the ambient temperature, and the solar radiation boundary is calculated by the Duffie solar radiation model.

To accurately simulate the temperature field of a bridge, the key is to consider the occlusion relationship of each surface of the bridge. For a common composite girder bridge, the main occlusion relationship in the model is the occlusion of the concrete girder overhanging section to the steel girder web, which is constantly changing during the simulation process. Since ABAQUS does not provide a radiation and temperature calculation module, to facilitate the modeling and batch calculation, this paper develops a simulation tools coded in Python language and Fortran subroutine.

\subsection{Model Validation}

The refined numerical model of temperature field is verified by the temperature field test in this paper. The results are shown in Figure 5 7. The simulated temperature distribution agrees well with the experimental distribution, which verifies the accuracy and reliability of the numerical model. 


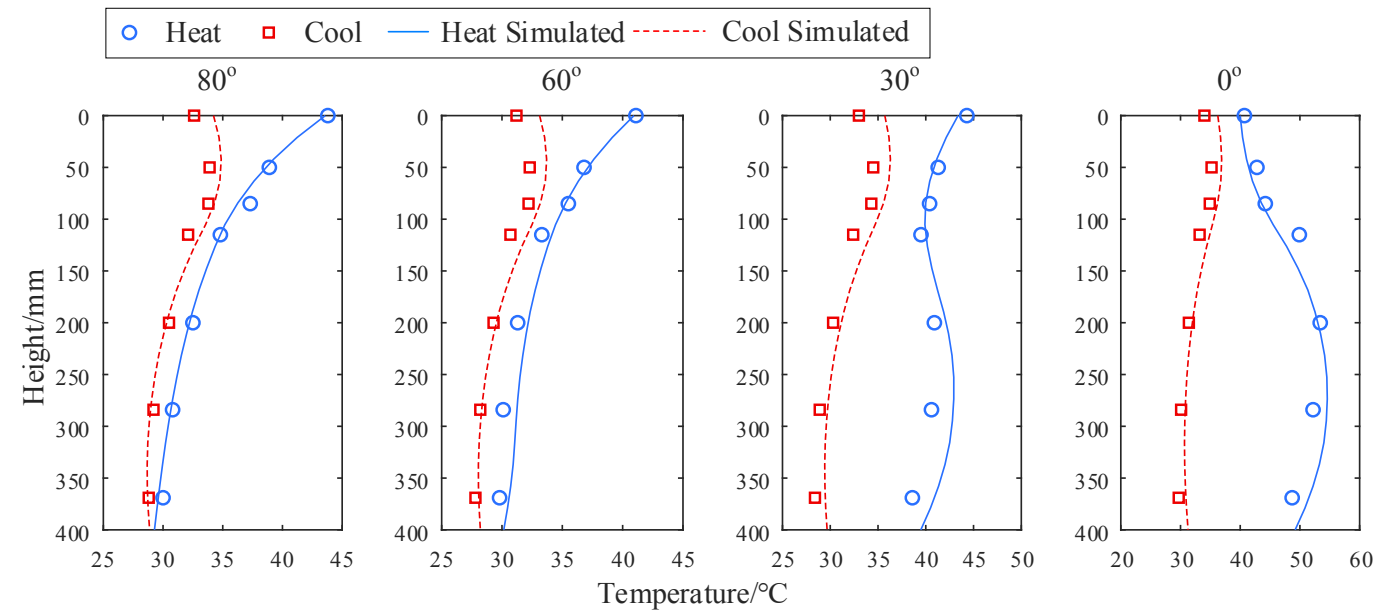

Figure 5 Comparison of simulated temperature and measured temperature in indoor test

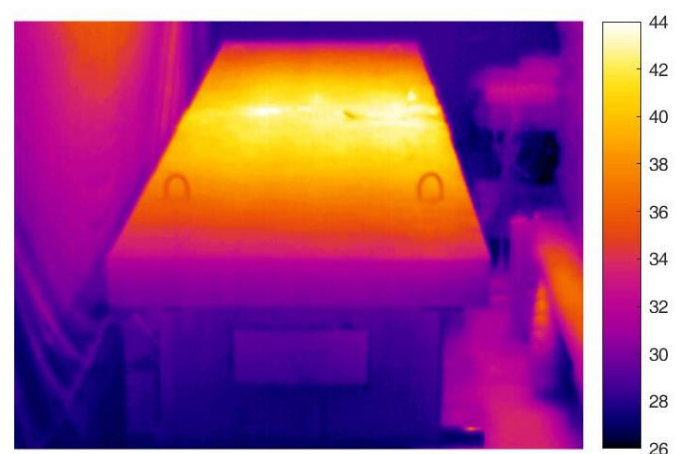

(a) thermal image

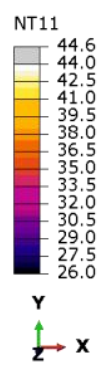

(b) simulated thermal field

Figure 6 Comparison of simulated temperature field and measured thermal image
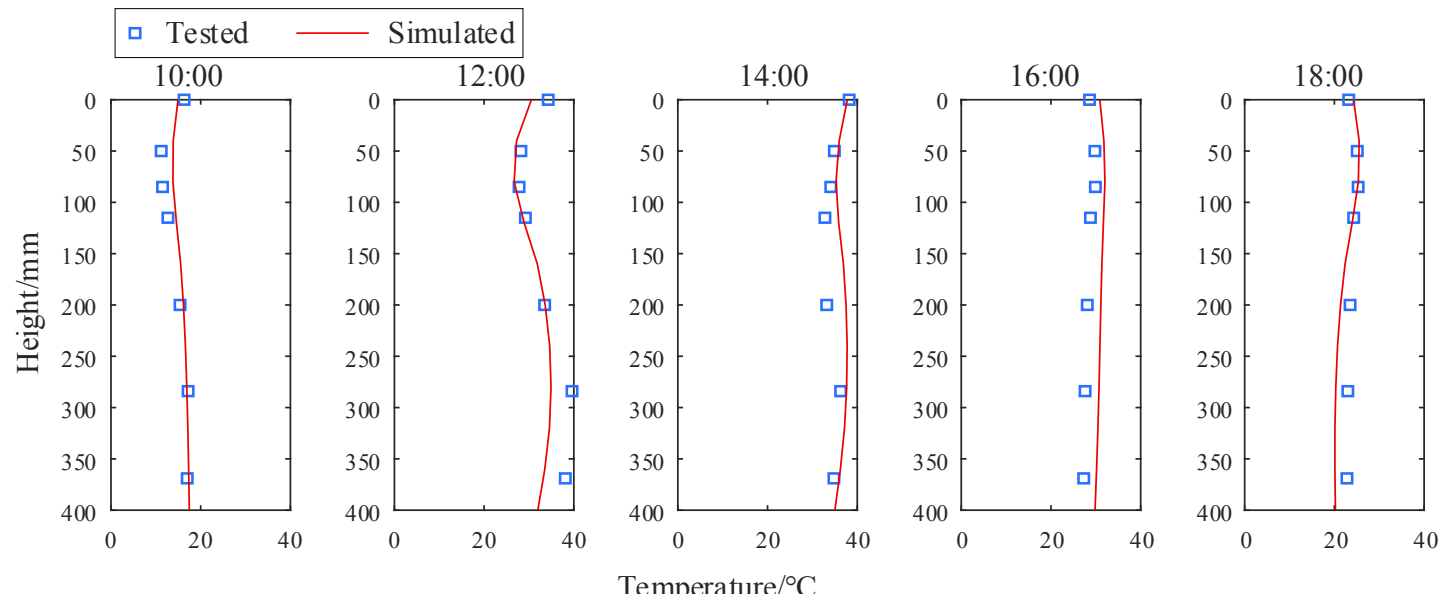

Figure 7 Comparison of simulated temperature and measured temperature in outdoor test

\section{The distribution law of the temperature field}

To simplify the temperature field calculation method and guide the engineering application, the temperature field test results and simulation results are analysed. Two typical temperature distribution modes of the composite beam are summarized, namely the vertical positive distribution mode and the vertical distribution mode. Both correspond to the positive temperature gradient load and the negative temperature gradient load in the bridge load specification.

\subsection{Positive distribution mode}

The positive distribution mode corresponds to the situation where the top surface of the composite beam is irradiated and the web is less irradiated, such as the working conditions of the radiation angles of $80^{\circ}$ and $60^{\circ}$ in the indoor test, and the sunshine heating stage of the outdoor test.

The vertical temperature distribution is characterized by the nonlinear decrease of the temperature field from top to bottom. This temperature distribution pattern produces a mechanism in which the concrete top surface 
of the composite beam receives solar radiation and transfers heat from top to bottom. Due to the high thermal conductivity of the steel beam, the temperature of the steel beam at the bottom of the test piece tends to be close to the ambient temperature.

The vertical positive temperature distribution can be simplified by using the third-order curve, as shown in Figure 8. Among them, $T_{1}$ is the representative value of the positive temperature gradient load, which should be comprehensively determined according to the climate statistics of the concerned area.

\subsection{Negative distribution mode}

The negative distribution mode corresponds to the heat dissipation stage, such as the heat dissipation condition in the indoor test and the temperature distribution at night in the outdoor test.
The vertical temperature distribution shows that the temperature of the concrete top and the steel bottom is the lowest, while the temperature inside the concrete slab is high. The mechanism of this temperature distribution pattern is after sunset (or when the indoor test is cooled), the ambient temperature is lower than that of the composite beam, and the concrete slab and the steel beam are radiated to the environment. The composite beam generates heat from the middle portion to the top of the plate and the bottom of the plate. Due to the good thermal conductivity of steel, the temperature will approach the ambient temperature faster, while the concrete heats up slowly and the temperature is relatively high.

The vertical positive temperature difference distribution can be simplified by using multiple straight lines, as shown in Figure 9. $T_{2}$ and $T_{3}$ are representative values of negative temperature gradient loads, which should be comprehensively determined according to the climate statistics of the concerned area.

Beam Radiation

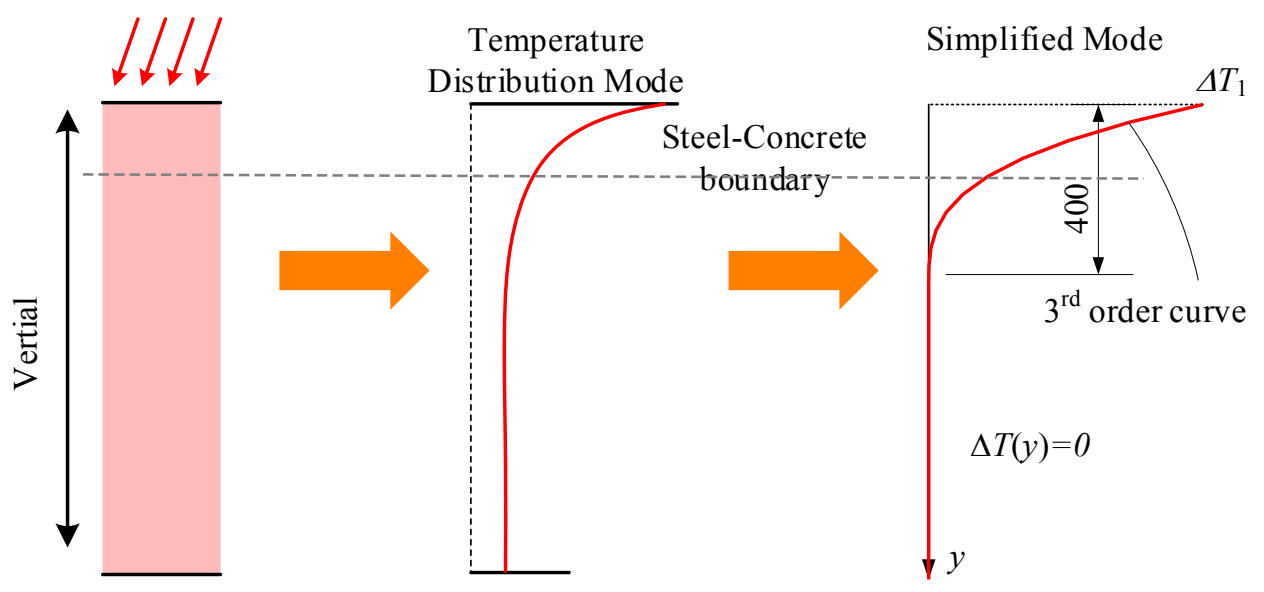

Figure 8 Positive temperature mode

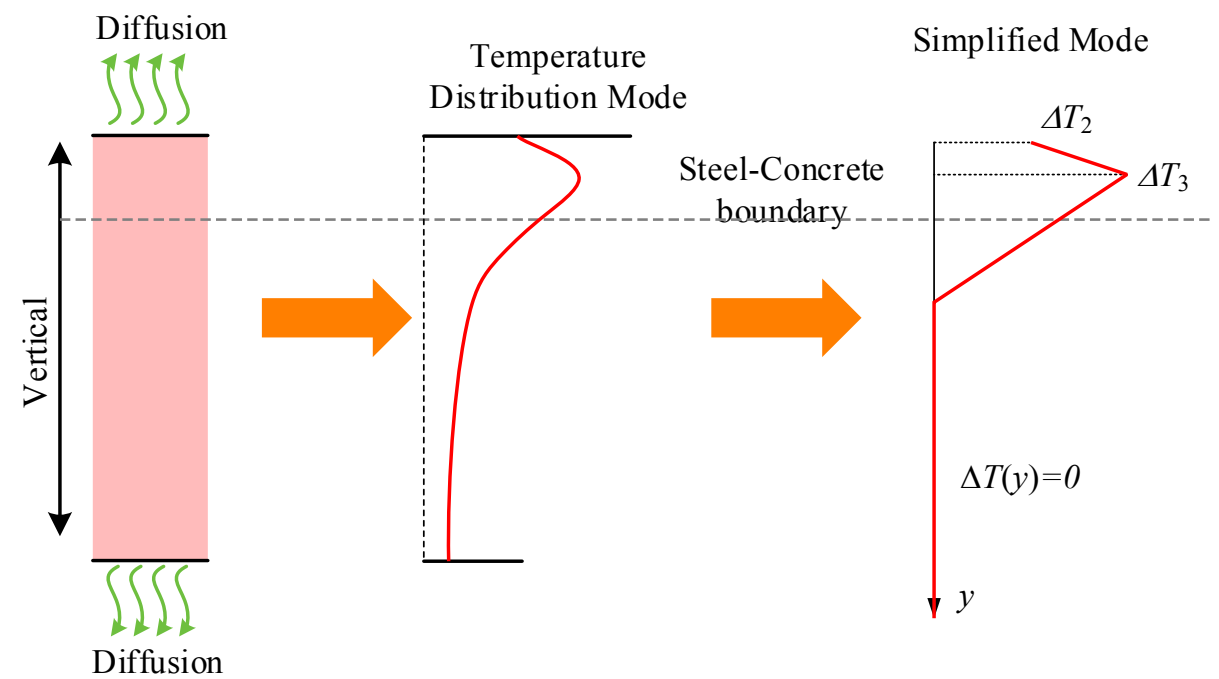

Figure 9 Negative temperature mode

\section{Conclusion}

The study of temperature field and temperature effect of steel-concrete composite bridges is of great significance to ensure the safety and durability of composite bridges. In this paper, the latest research progress in the field of composite bridge temperature field is introduced, including the composite bridge temperature field test 
technology, the bridge temperature fine finite element model, and the temperature distribution laws. The proposed finite element model of temperature field can accurately and reliably simulate the solar temperature field for composite bridges. The proposed two composite bridge temperature distribution modes can be used as reference for bridge designers.

\section{Acknowledgement}

The authors would like to thank the fund of the National Natural Science Foundation of China (51478245).

\section{References}

1. Fu, H.C., Ng, S.F. and Cheung, M.S., (1990) Thermal behavior of composite bridges. Journal of Structural Engineering, 116(12): 3302-3323.

2. Kennedy, J.B. and Soliman M.H. (1987) Temperature distribution in composite bridges. Journal of Structural Engineering., 113(3): 475-482.

3. Dilger, W.H., Ghali, A., Chan, M., Cheung, M.S. and Maes, M.A. (1983) Temperature stresses in composite box girder bridges. Journal of Structural Engineering, 109(6): 1460-1478.

4. Su, J.H. Duan, S.J. (2012) Research on the solar temperature field of double-side steel-concrete composite box beam. Journal of Shijiazhuang Tiedao University, 2012: 6-10. (in Chinese)

5. Sun, G.C. Guan, R.C. Jiang, Y.M. Mou, C.L. Huo, C.Y. Xu, F. (2006). Research of the segmental temperature distribution of steel-concrete composite beam. Engineering Mechanics, 23(11): 122-127. (in Chinese)

6. Duffie, J.A. and Beckman W.A. (2013) Solar engineering of thermal processes. John Wiley \& Sons. 